\title{
R\&D Progress in Wireless Communications in China - A Review on Chinese High-Tech R\&D Programs for Wireless Technology
}

Ke GONG ${ }^{\dagger a)}$ and Jing WANG ${ }^{\dagger \dagger}$, Nonmembers

\begin{abstract}
SUMMARY China has experienced fast growth in mobile communications. Now, China is the world largest mobile communication country with about 500 million users. Wide applications of mobile communications are giving strong pull to the research and development on the broadband wireless communication technology to meet the fast growing demand for high speed access into the information infrastructure. This makes the R\&D on wireless technology play great role in the Chinese High-Tech program. This paper will review the key project - FuTURE (Future Technology for Universal Radio Environment) - development of the 863 program, which represents the Chinese efforts towards IMT-advance. Taking some works done in the Tsinghua National Laboratory for Information Science and Technology as examples, the paper will show what has been made in China on the broadband wireless technology, including the trial network in Shanghai.

key words: China's hightech program, wireless communication, distributed cellular system, interleave division multi access, constellation overlapping
\end{abstract}

\section{Introduction}

Twenty years ago, 4 well-known Chinese Scientists wrote to Mr. Deng Xiaoping, the leader of China, proposing to implement a national high-tech $R \& D$ program to meet the technological challenges raised by the worldwide scientific and technological revolution. The proposal had been soon accepted by the Chinese government and the Chinese hightech $R \& D$ program has been put into practice, it is known as " 863 " program to memorize the historical decision made by Deng Xiaoping in March, 1986.

It is to note that the 863-program is not just to support some technical R\&D projects, but also an important reform of the Chinese R\&D system. It has 3 distinguished characteristics:

(1) Focusing on the selected high-tech areas, such as Information Technology, Biological and Agricultural technology, technology for advanced manufacturing and automation, material technology, energy and environmental technology, etc.;

(2) Opening to all researchers in all research institutes, universities, industry and military for application;

(3) Steered by experts group, in terms of selecting proper projects from thousands of applications and determining the amount of money assigned to each approved projects.

Now, the 863-program is the largest Chinese govern-

\section{Manuscript received May 13, 2007.}

${ }^{\dagger}$ The author is with Tianjin University, Tianjin, China 300032.

${ }^{\dagger}$ The author is with Tsinghua National Laboratory for Information Science and Technology, Beijing, China 100084.

a)E-mail: gongk@tju.edu.cn

DOI: 10.1093/ietcom/e90-b.9.2208 mental program for $\mathrm{R} \& \mathrm{D}$, with its budget of billions of RMB in the period of 2006-2010.

Though IT has been selected as one of the selected of the 863-program, the telecommunication technology had not been included until 1992!

In 1987, in a south province near to Hong Kong, the public mobile telecom system, using TACS standard, had been implemented. By the end of that year, there were hundreds of subscribers using the handset cost 20 times higher than the China's GDP per capital in that year or 50 times higher than yearly income of ordinary workers. Nobody could imagine, only in a few years, mobile communication has revolutionary changed the Chinese telecom system and the market by a penetration of more than 30 percent of the total population.

Entering the 1990's, China realized that it must pay more attention to the information revolution, especially to catch up of world's pace by taking the opportunity of "digitalization" of telecommunication technology, so that "telecommunication," as a special area called "Tele-863," had been added into 863 program with emphasis on digital mobile communication and high speed optical communication.

Reviewing to the 15 years of "Tele-863" program, in terms of mobile communication, it could be divided into two phases. In the first 10 years, the program could be considered as " $3 G$ "-oriented one, while in recent years which has become "B3G" (beyond 3G) - oriented. The latter is represented by the "FuTURE" project.

\section{2. “FuTURE” Project}

Experienced the fast growth of mobile communication, there is strong pull to the wireless technology by the wideband or high-speed applications. And, there are notable trends of the merging of the next generation of internet, the next generation of optic communication and the next generation of wireless communications. Therefore, in recent years, the IT sub-program has been focusing on (1) Next generation of networks, especially the IPv6 based next generation of internet; (2) Optical technology for internet with multi-wavelength environment (O-TIME project) and (3) Future technology for universal radio environment (FuTURE-project). The last one, FuTURE project, is a $R \& D$ project for wireless technique with a governmental budget about 30 million USD, aiming to develop a B3G trial system with innovated techniques to meet the requirements 
of the evolution from $3 \mathrm{G}$ to access to the IP-based new generation of core networks [8]. It is to note that a distinguished feature of FuTURE project is international collaboration. The main topics of FuTURE (2003-2005) are:

- B3G test system and key techniques

- B3G RTT assessment platform

- B3G applications, standards and measurement

- Self-organized network based on WLAN \& 3G

- New type of antenna and diversity techniques

- Ultra-Wide Band (UWB) transmission techniques

- Emerging wireless techniques

* THz techniques

* Cognitive radio techniques

* Novel access architecture to public mobile network

* EMC for multiple wireless systems with shared frequency spectrum

Thousands of researchers including students from many universities as well as research institutes and industrial companies are involved in the FuTURE project, and notable progresses have been made by the years of afford. A field test system of B3G has been established in Shanghai, which integrated many newly developed techniques. Hundreds of technical papers have been published and more than 100 invention patents have been filed along with about 100 recommendations have been proposed to international standardization organizations (3GPP, 3GPP2, etc.). Furthermore, a number of new techniques about MIMO, UWB, OFDM, etc., have been developed, which will play important roles in the next phase of FuTURE in the coming 5 years.

\section{Some Examples of FuTURE Project Carried by TNList}

TNList stands for Tsinghua National Laboratory for Information Science and Technology, which is one of the 6 established National Laboratories in China. As an important member of the FuTURE project, TNList has carried out a number of topics of the project and made distinguished contributions to the FuTURE. The followings are 3 examples of TNList's works aiming at breakthrough the limitation of power and bandwidth to the high speed and high capacity wireless communications [1].

\subsection{Distributed Wireless Cellular System (DWCS) and MIMO}

Figure 1 shows the proposed new type of network architecture, in which a mobile user is not served by one base-station as usual in a conventional cellular system, but by a number of "nodes" around it, where the Node-A is just an antenna only while Node-B has signal processing functions. A virtual Node-B (like a base-station) consists of a number of Node A and B, serving a virtual cell. This kind of network, named as Distributed Wireless Cellular System (DWCS), is a user (mobile) centralized cellular system with user (mobile) centralized processing and coverage, while the conven-

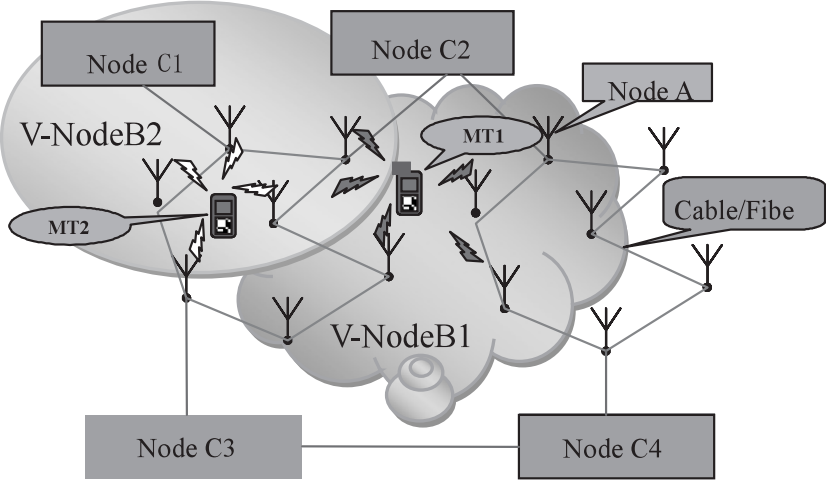

Fig. 1 The configuration of Distributed Wireless Cellular System (DWCS).

tional cellular system is base-station centralized system [2].

Supported by RoF (Radio over Fiber) technology, antennas (Note A) could be distributed to build a macrodiversity environment to every user with low cost and lower power per square meter.

The performance comparison of DWCS and MIMO are shown in Fig. 2, it is found that the MIMO increases the peak capacity while the DWCS increases the base-plat capacity. Therefore, the DWCS and MIMO could be combined and jointly optimized by balancing the elements between them to meet the different requirements of capacity [3].

\subsection{Constellation Overlapping Technique}

Besides the "spatial domain" technique of DWCS and MIMO, TNList has made effort in "power domain" to further increase the network capacity, called "constellation overlapping" technique.

In the wireless cell, different users are in different positions with different channel conditions. The traditional design of cellular network is to ensure the worst case performance. The adaptive modulation technique is to use TDM scheme to serve different users in different channel conditions with different modulation schemes, e.g. user $A$ and $B$ are in different position with $\mathrm{S} / \mathrm{N}$ of 30 and $10 \mathrm{~dB}$ each (Fig. 3), so that they could be served by 64QAM and BPSK scheme respectively to increase the overall transmission rate by exploring the potential of the channel. Comparing to the conventional BPSK design with throughput efficiency of $1 \mathrm{bit} / \mathrm{s} / \mathrm{Hz}$, the TDM adaptive modulation could increase the throughput efficiency to $(1+6) / 2=3.5 \mathrm{bit} / \mathrm{s} / \mathrm{Hz}$. However, instead of TDM based adaptive modulation, TNList proposed a constellation overlapping technique, which uses overlapped 16QAM and BPSK to serve user A and B respectively, and could get a throughput efficiency of $(1+4)=5 \mathrm{bit} / \mathrm{s} / \mathrm{Hz}$ (Fig. 4). Figures 5 and 6 show the received signal constellations by user $\mathrm{A}$ and $\mathrm{B}$ respectively. It is apparent that the constellation overlapping modulation technique (COM) could be a multiple definition technology in digital broadcasting systems and a power multiplexing technology in downlink transmissions. 


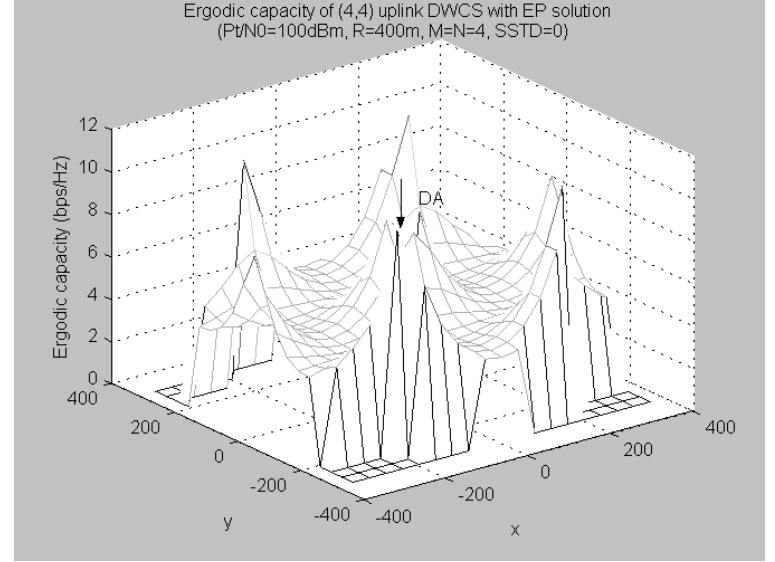

(a)

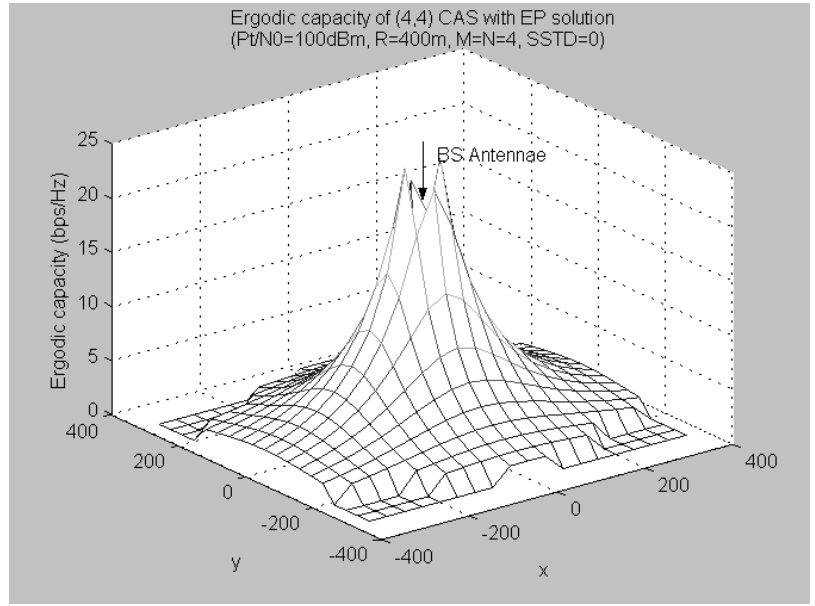

(b)

Fig. 2 The effects of (a) DWCS and (b) MINO on system capacity.

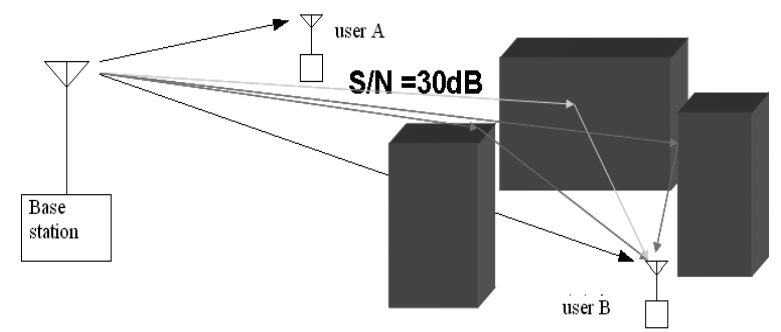

\section{$S / N=10 d B$}

Fig.3 Illustration of different user with different channel condition.

3.3 Interleaving Pattern Division Multiple Access Technique (IDMA)

Another "code domain" technique called "interleaving pattern division multiple access (IDMA)" has been introduced by TNList, as shown in Fig. 7, the data from different users are processed by different interleaving schemes, and this outperforms the CDMA in high level modulations of OFDM (Fig. 8). It is considered that IDMA could be a good solution for the same frequency reuse among adjacent cells, or a

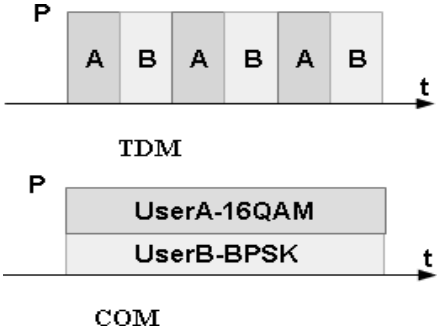

Fig. 4 Illustration of TDM (top) and COM (bottom) scheme.

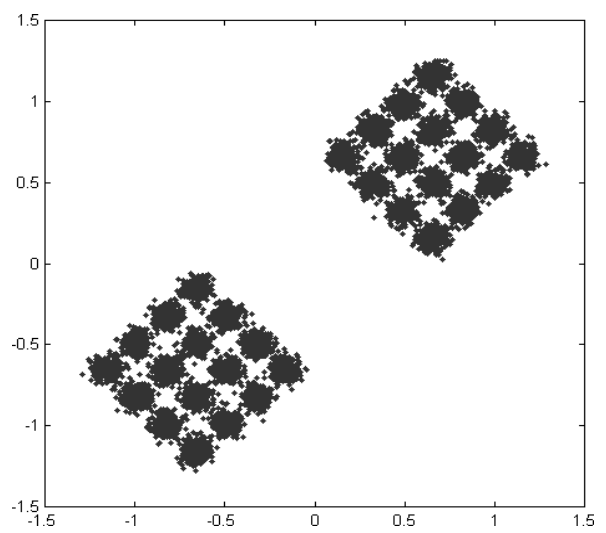

Fig. 5 Illustration of received constellation by user A.

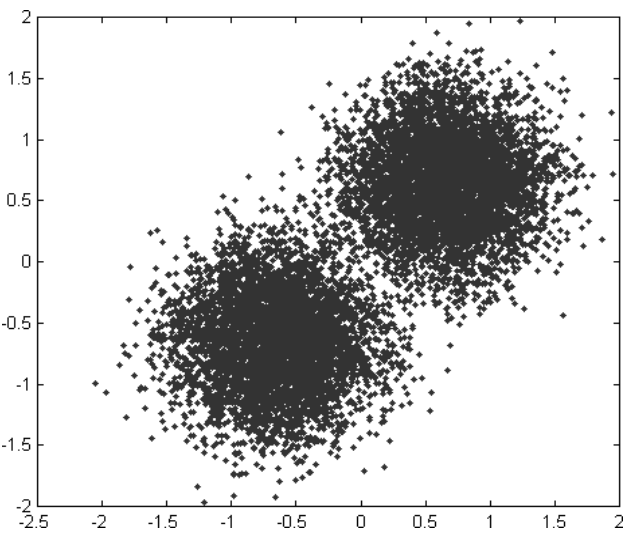

Fig. 6 Illustration of received constellation by user B.

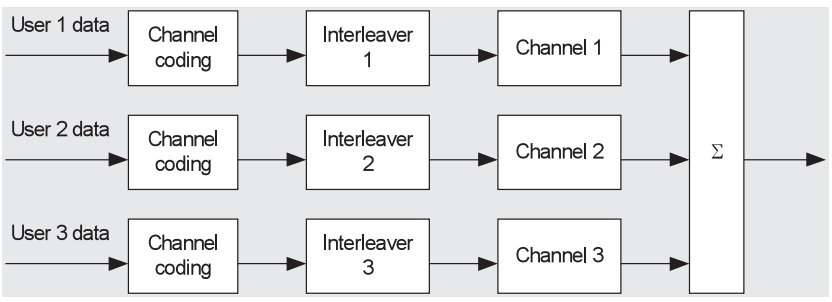

Fig. 7 The interleaving pattern division multiple access technique.

multi-cell frequency reuse technique. 


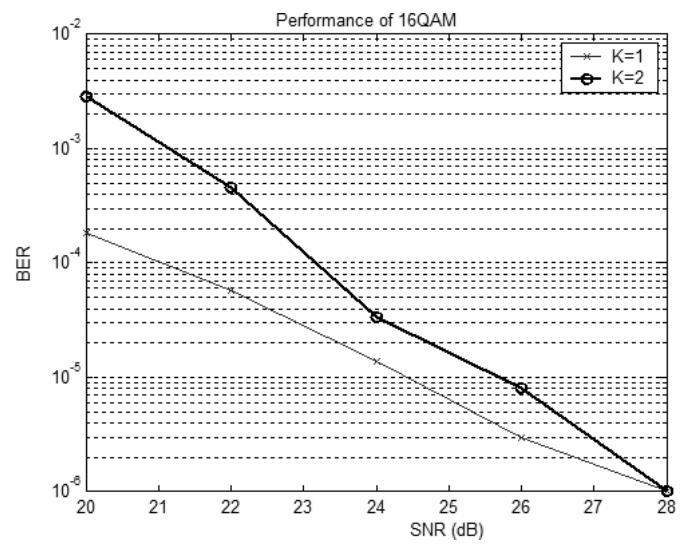

Fig. 8 IDMA performance comparison of one and two users.

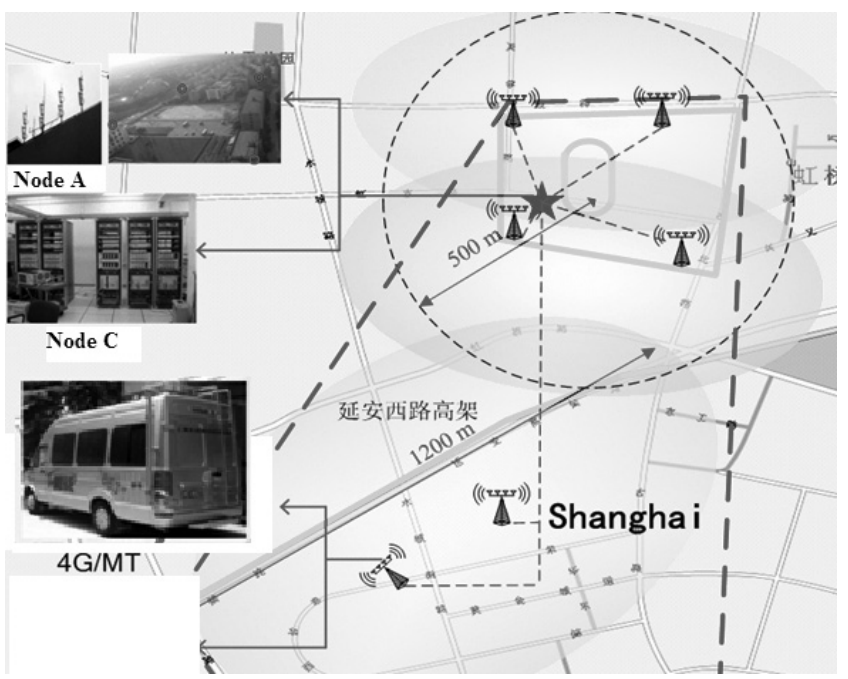

Fig. 9 Illustration of the Shanghai testbed of FUTURE.

\section{The Testbed of FuTURE}

To demonstrate the developed techniques in the FuTURE project, a trial network has been established in Shanghai. The trial network consists of 6 access-points making 3 cells covering both urban and suburban freeways, each cell covers $0.5-1 \mathrm{~km}$ in radii, and there are 6 terminals mounted in vehicles with moving speed from 0 to $120 \mathrm{~km}$ per hour. The carrier used is in $3.5 \mathrm{GHz}$ band with a bandwidth of $20 \mathrm{MHz}$. The modulation scheme is General multi-carrier for uplink and OFDM for downlink. $8 \times 4$ and $4 \times 4$ MIMO are used. The data rate is $100 \mathrm{Mbps}$. Demonstrated service includes IP based SDTV and HDTV program, real-time HD video, video conferencing, Voice over IP phone, web browse and FTP, etc.

Figure 9 illustrates the test bed in Shanghai. And Fig. 10 shows the improved performance by DWCS technique.

In the test bed, the techniques developed in the $\mathrm{Fu}$ TURE project, such as DWCS, IDMA, as well as adaptive MIMO and double-turbo iterative receiver, have been well

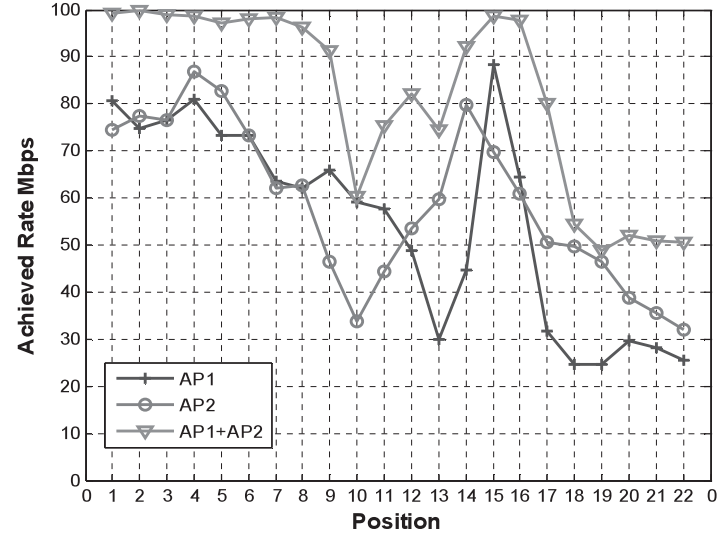

Fig. 10 Illustration of the approved performance by adopting DWCS technique (using AP1+AP2 instead of using AP1 or AP2).

integrated and shown good performances.

\section{Conclusion Remarks}

With the help of 863-program, China is now playing an ever-active role in the world high tech development stage. FuTURE is the core project of Chinese wireless technique research and development, which is also a platform for international collaborations. Working closely with partners in China and in the world, TNList has been making great contributions to the FuTURE project with many innovative techniques in the network, transmission as well as physical layers. The further efforts of the FuTURE project (20062010) will focusing on IMT-advance relevant technology and low-cost high-speed short range wireless access system, including varies techniques of system architectures, networking structures and protocols, novel terminals and human-machine interfaces, open and reconfigurable hardware platforms, pervasive radio communications, etc.

\section{Acknowledgments}

The authors thank Prof. YOU Xiaohu of Southeast University, Nanking, China, Prof. ZHANG Ping of Beijing University of Post and Telecommunications, Beijing, China, and other partners from University of Science and Technology China, Shanghai Jiaotong University, Xian Jiaotong University, Xidian University, University of Electronic Science and Technology China, etc., and Huawei Technology for the fruitful collaboration in the past years for the FuTURE project.

References

[1] S. Zhou, Y. Li, M. Zhao, X. Xu, J. Wang, and Y. Yao, "Novel techniques to improve downlink multiple access capacity for beyond 3G," IEEE Commun. Mag., vol.43, no.1, pp.61-69, Jan. 2005.

[2] S. Zhou, M. Zhao, X. Xu, J. Wang, and Y. Yao, "Distributed wireless communication system: A new architecture for future public wireless access," IEEE Commun. Mag., vol.41, no.3, pp.108-113, March 2003. 
[3] L. Dai, S.D. Zhou, and Y. Yao, "Effects of macrodiversity and microdiversity on CDMA forward-link capacity," IEICE Trans. Commun., vol.E85-B, no.4, pp.748-757, April 2002.

[4] D. Wang, B. Han, J. Zhao, X. Gao, and X. You, "Channel estimation algorithms for broadband MIMO-OFDM sparse channel," 14th IEEE Proc. Personal, Indoor and Mobile Radio Communications 2003, vol.2, pp.1929-1933, Sept. 2003.

[5] Z. Jiang, J. Wu, X. Zhao, and X. You, "A model for radio access unit selection in distributed radio system," 6th International Conference on ITS Telecommunications Proceedings 2006, pp.834-837, June 2006.

[6] G. Liu, X. Liu, Y. Wang, and P. Zhang, "Efficient interference based RRM for speech service in FuTURE TDD system," IEEE International Symposium on Microwave, Antenna, Propagation and EMC Technologies for Wireless Communications 2005, vol.2, pp.14521455, Aug. 2005.

[7] G. Liu, J. Zhang, and P. Zhang, "Further vision on TD-SCDMA evolution," 2005 Asia-Pacific Conference on Communications, pp.143147, Oct. 2005.

[8] X.-H. Yu, G. Chen, M. Chen, and X. Gao, "The FuTURE project in China," IEEE Commun. Mag., vol.43, no.1, pp.70-75, Jan. 2005.

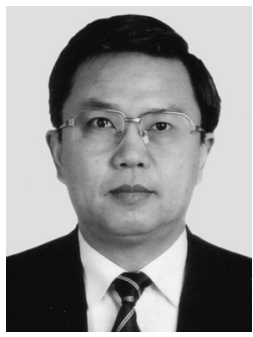

Ke Gong received the Bachelor's and Doctoral Degrees in technical science from Beijing Institute of Technology (BIT), Beijing, China, in 1982 and Technical University of Graz, Austria, in November 1986, respectively. From 1987-2006, he worked in Tsinghua University on wireless channel characteristics, broadband wireless access, antenna, and terrestrial Digital TV transmission, etc. He is now with Tianjin University and vice chairman of China Institute of Communications (CIC), vice chairman of Chinese Institution of Electronics (CIE).

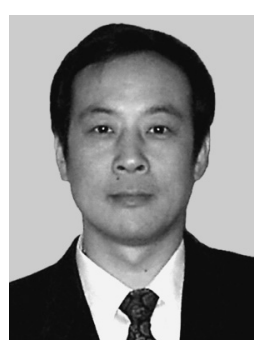

Jing Wang received the B.S. and M.S. degree in electronic engineering from Tsinghua University, Beijing China in 1983 and 1986 respectively. He has been on the Faculty at the Tsinghua University since 1986 . He currently is a professor of the School of Information Science and Technology. His research interests are in the area of wireless communications, including systems of $3 \mathrm{G}$ and $\mathrm{B} 3 \mathrm{G} / 4 \mathrm{G}$, channel coding, multiuser detection, and MIMO technologies. He is a member of the coordination Group of the FuTURE Program. He is also a member of Radio Communication Committee of CIC and a senior member of CIE. 